\title{
Renforts ordinaires ou précontraints sur tuyaux et conduites forcées, tuyaux frettés et autofrettés
}

\section{Ordinary and prestressed stiffeners for pipes and penstocks}

PAR
Claude TASCHER

INGÉNIEUR DES ARTS ET MANUFAGTURES
ET

L'étude est relative aux contraintes produites par la pression interne ou externe, et par un effort longitudinal quelconque dans un tugan muni de renforts circulaires équidistants.

On distingue :

- Le cas de renforts suffisamment rapprochés pour que leur interaction ne soit pas négligeable:

- Le cas de renforts isolés;

- Le cas de renforts précontraints.

Dans ces divers cas, on prend en considération la forme et la largeur dn renfort. Les contraintes ainsi calculées dans le renfort et dans la tôle du tuyau, tiennent comple de toutes les modifications apportées par la précontrainte ou par un effort longitudinal, en particulier par l'effet Poisson de la pression.

Enfin, l'étude est étendue aux tuyaux frettés $\dot{a}$ chaud et aux tubaux autofrettés, qui ne constituent qu'un cas particulier de la théorie précédente.

Dans la construction courante des tuyaux, et particulièrement des tuyaux de grand diamètre et de faible épaisseur, l'ingénieur est souvent amené à fixer, sur un tuyau cylindrique, un ou plusieurs renforts constitués par exemple par un fer plat, une cornière, un profilé quelconque, la liaison entre renfort et tuyau étant assurée par une soudure ou par simple contact.

Ce renfort aura pour but, par exemple, d'assurer la stabilité du tuyau sous l'effet d'une pression extérieure, d'augmenter l'inertie de la section à l'emplacement d'un supportage, de permettre l'ancrage du tuyau dans une maconnerie.

Si les renforts sont très rapprochés, leur but peut être simplement de constituer un complexe

\author{
Monioue REY-JOUVIN \\ INGŔNIEUR DE L'ÉCOLE POLYTEGHNIQUE FÜMININE
}

This study relates to the stresses consed by internal and external pressures and by a longitadinal load in a pipe fitled with equally spaced circular stiffeners. The following cases are considered:

1. Stiffeners so closely spaced that their interaction cannot be neglected;

2. Isolated stiffeners;

3. Prestressed stiffeners.

The shape and width of the stiflener are considered in all three cases. The stiffener and pipe shell stresses thus calculated allow for all the modifications resulting from the prestressing or from a longitudinal load, especially as a result of the Poisson effect of the pressure.

Pipes hooped by hot-shrinking and self-hooped ("autofretted") pipes-which are a special case of the theory-are also considered in the study.

tôle-renfort, précontraint ou non, capable de résister à la pression intérieure.

Nous nous proposons, dans ce qui suit, d'étudier les contraintes créćes, dans la tôle du tuyau et dans le renfort par une pression intérieure ou extérieure au tuyau. Il s'agit donc d'une symétrie circonférencielle totale.

La présence des renforts modifie, sur une certaine longueur, la dilatation radiale du tuyau sous l'effet de la pression, et, simultanément, induit des contraintes longitudinales dues à la flexion de la tôle.

Notre étude se bornera à une structure longitudinale périodique et s'ćtendant théoriquement, sur une longueur infinie. Il s'agira donc, dans le 
cas le plus général, de renforts ou groupes de renforts régulièrement espacés; on conçoit facilement que, si cet espacement est suffisamment faible, chaque renfort superpose, dans une cerlaine zone, son action à celle des renforts voisins. Le cas d'un renfort isolé ou considérè comme tel - espacement suffisamment grand, -- se déduira très simplement du cas général.

Nous nous proposons parallèlement, d'étudier l'influence d'un effort longitudinal sur les contraintes ainsi trouvées.
Enfin, dans ce qui suit, nous supposerons :

$1^{\circ}$ Que la tòle du tuyau et le métal du renfort ont le même module d'élasticité:

2" Que le rapport $e / \mathrm{R}$ de l'épaisseur du tuyau à son rayon intérieur est suffisamment faible pour qu'on puisse appliquer la théorie des coques minces, ce qui revient à négliger l'effet Lamé.

\section{A. - ÉTUDE PRELIMINAIRE DE LA FLËCHE RADIALE PRODUITE PAR DES FORCES EGALES ET ËQUIDISTANTES REPARTIES ANNULAIREMENT SUR UN TUYAU}

\section{A.1 - Flèche produite au droit de la force centrale par $2 n+1$ forces.}

Soit P la valeur de la force par unité de longueur circonférencielle, $l=$ l'espacement des forces. On sait (1) que $\mathrm{P}$ produit, à une distance $x$ de son cercle d'action une flèche :

avec :

$$
\mathrm{W}=\frac{\mathrm{PR}^{2} \beta}{2 e \mathrm{E}} ?(\beta x)
$$

et

$$
\beta^{4}=\frac{3\left(1-y^{2}\right)}{R^{2} e^{2}}
$$

$$
\varphi(\beta x)=e^{-\beta x}(\sin \beta x+\cos \beta x)
$$

Si on prend l'origine des $x$ au droit de la force centrale et que l'on considère d'abord les forces $0,1,2 \ldots n$, de la moitié droite du tuyau, on écrira :

$$
\begin{aligned}
& \frac{\mathrm{P}}{2} \text { appliqué en } 0 \text { crée en } 0 \text { une flèche } \mathrm{W}=\frac{1}{2} \frac{\mathrm{PR}^{2} \beta}{2 e \mathrm{E}} \varphi(\beta .0) \\
& \mathrm{P} \text { appliqué en } 1 \text { crée en } 0 \text { une flèche } \mathrm{W}=\frac{\mathrm{PR}^{2} \beta}{2 e \mathrm{E}} \varphi(\beta . l) \\
& \mathrm{P} \text { appliqué en } 2 \text { crée en } 0 \text { une flèche } \mathrm{W}=\frac{\mathrm{PR}^{2} \beta}{2 e \mathrm{E}} \varphi(\beta .2 l)
\end{aligned}
$$

$\mathrm{P}$ appliqué en $n$ crée en 0 une flèche $\mathrm{W}=\frac{\mathrm{PR}^{2} \beta}{2 e \mathrm{E}} \varphi(\beta . n l)$

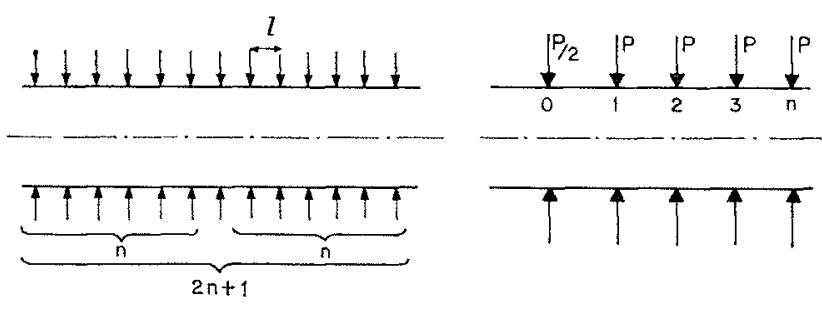

FiG. 1

(1) Timoshenio : Plaques et Coques, p. 379. 
La superposition de ces divers états donnera la flèche, en 0 , créée par la moitié droite du tuyau, soit, après développement et transformation des diverses sommations :

$$
\mathrm{W}=\frac{\mathrm{PR}^{2} \beta}{2 e \mathrm{E}}
$$$$
\left[\frac{e^{-\beta l(n+2)}(\cos \beta l n+\sin \beta l n)-e^{-\beta l(n+1)}[\cos \beta l(n+1)+\sin \beta l(n+1)]-e^{-\beta l}(\cos \beta l-\sin \beta l)+1}{1+e^{-2 \beta l}-2 e^{-\beta l} \cos \beta l}-\frac{1}{2}\right]
$$

La flèche totale en 0 , créée par le $2 n+1$ forces, sera donc :

$$
\mathrm{W}=\frac{\mathrm{PR}^{2} \beta}{2 e \mathrm{E}}
$$

$\times\left[\frac{2 e^{-\beta l(n+2)}(\cos \beta l n+\sin \beta l n)-2 e^{-\beta l(n+1)}[\cos \beta l(n+1)+\sin \beta l(n+1)]-2 e^{-\beta l}(\cos \beta l-\sin \beta l)}{1+e^{-2 \beta l}-2 e^{-\beta l} \cos \beta l}-1\right]$

\section{A.2 - Flèche produite par $2 \boldsymbol{n}$ forces dans une section comprise entre les deux forces centrales.}

Si on prend l'origine des $x$ au droit de la section $\mathrm{S}$ considérée, et que l'on considère d'abord les forces $1,2, \ldots n$, de la moitié droite du tuyau, on écrira :

$P$ appliqué en 1 crée en $S$ une flèche $W=\frac{P^{2} \beta}{2 e E} \bullet[\beta(0 \times l+\lambda)]$

$\mathrm{P}$ appliqué en 2 crée en $\mathrm{S}$ une flèche $\mathrm{W}=\frac{\mathrm{PR}^{2} \beta}{2 e \mathrm{E}} \cdot[\beta(1 \times l+\lambda)]$

$\mathrm{P}$ appliqué en $n$ crée en $\mathrm{S}$ une flèche $\mathrm{W}=\frac{\mathrm{PR}^{2} \beta}{2 e \mathrm{E}} \varphi[\beta((n-1) l+\lambda)]$
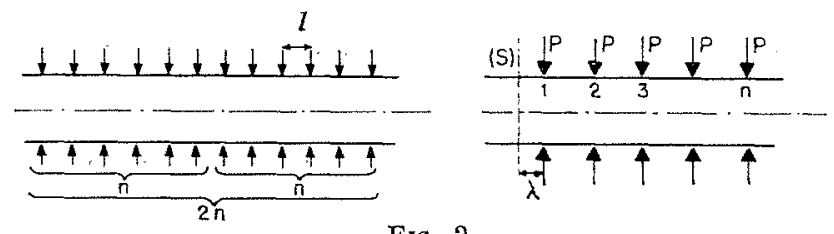

FIG. 2

La superposition de ces divers états donnera la flèche en $S$ créée par les $n$ forces situées à droite de la section, soit après développement :

$$
\begin{aligned}
& \mathrm{W}=\frac{\mathrm{PR}^{2 \beta}}{2 e \mathrm{E}} \frac{e^{-\beta \lambda}}{1+e^{-2 \beta l}-2 e^{-\beta l} \cos \beta l}\left\{e^{-\beta l(n+1)}[\cos (\beta l(\mathrm{n}-1)+\beta \lambda)+\sin (\beta l(n-1)+\beta \lambda)]\right. \\
& -e^{-\beta \ln }[\cos (\beta \ln +\beta \lambda)+\sin (\beta \ln +\beta \lambda)]-e^{-\beta l}[\cos \beta(l-\lambda)-\sin \beta(l-\lambda)]+\cos \beta \lambda+\sin \beta \lambda \text { ! }
\end{aligned}
$$

L'action des forces situces à gauche de la section s'obtiendra en remplaçant, dans l'expression précédente, $\lambda$ par $l-\lambda$. La superposition de ces deux états donnera finalement la flèche en $S$ :

$$
\begin{aligned}
& \mathrm{W}=\frac{\mathrm{PR}^{2} \beta}{2 e \mathrm{E}} \frac{1}{1+e^{-2 \beta l}-2 e^{-\beta l} \cos \beta l}\left\{e^{-\beta[l(n+1)+\lambda]}[\cos \beta(I(n-1)+\lambda)+\sin \beta(l(n-1)+\lambda)]\right. \\
& -e^{-\beta(n+\lambda)}[\cos \beta(\ln +\lambda)+\sin \beta(\ln +\lambda)]-e^{-\beta(l+\lambda)}[\cos \beta(l-\lambda)-\sin \beta(l-\lambda)] \\
& +e^{-\beta \lambda}(\cos \beta \lambda+\sin \beta \lambda)+e^{-\beta[l(n+2)-\lambda]}[\cos \beta(\ln -\lambda)+\sin \beta(\ln -\lambda)] \\
& -e^{-\beta[l(n+1)-\lambda]}[\cos \beta(l(n+1)-\lambda)+\sin \beta(l(n+1)-\lambda)] \\
& -e^{-\beta(2 l-\lambda)}(\cos \beta \lambda-\sin \beta \lambda)+e^{-\beta(l-\lambda)}[\cos \beta(l-\lambda)+\sin \beta(l-\lambda)] \text { ! }
\end{aligned}
$$




\section{A.3 - Flèche produite par un nombre infini de forces.}

Si l'on rend $n$ infini dans l'expression [1]:

avec

$$
\mathrm{W} \text { sous la force }=\frac{\mathrm{PR}^{2} \beta}{2 e \mathrm{E}}\left[\frac{1-e^{-2 \beta l}+2 e^{-\beta l} \sin \beta l}{1+e^{-2 \beta l}-2 e^{-\beta l} \cos \beta l}\right]=\frac{\mathrm{PR}^{2} \beta}{2 e \mathrm{E}} \mathrm{K}(\beta l)
$$

$$
K(\beta l)=\frac{\operatorname{sh} \beta l+\sin \beta l}{\operatorname{ch} \beta l-\cos \beta l}
$$

De la même manière l'expression [2] devient:

$$
\begin{aligned}
\mathrm{W} \text { entre } 2 \text { forces } & =\frac{\mathrm{PR}^{2} \beta}{2 e \mathrm{E}} \frac{1}{1+e^{-2 \beta l}-2 e^{-\beta l} \cos \beta l} \\
\qquad & {\left[\begin{array}{c}
e^{-\beta(l-\lambda)}[\cos \beta(l-\lambda)+\sin \beta(l-\lambda)]-e^{-\beta(l+\lambda)}[\cos \beta(l-\lambda) \\
-\sin \beta(l-\lambda)]+e^{-\beta \lambda}(\cos \beta \lambda+\sin \beta \lambda)-e^{-\beta(2 l-\lambda)}(\cos \beta \lambda-\sin \beta \lambda)
\end{array}\right] } \\
& =\frac{\mathrm{PR}^{2} \beta}{2} \frac{\mathrm{e}}{\mathrm{E}} \mathrm{H}(\beta \lambda)
\end{aligned}
$$

avec $H(\beta \lambda)=\frac{\operatorname{sh} \beta \lambda \cos \beta(l-\lambda)+\operatorname{ch} \beta \lambda \sin \beta(l-\lambda)+\operatorname{sh} \beta(l-\lambda) \cos \beta \lambda+\operatorname{ch} \beta(l-\lambda) \sin \beta \lambda}{\operatorname{ch} \beta l-\cos \beta l}$

On vérifie facilement que lorsque $\lambda=0$ ou bien $\lambda=l, H(\beta \lambda)$ devient égal à $K(\beta l)$.

\section{B. - ÉTUDE D'UN TUYAU RENFORCÉ \\ PAR UNE INFINITE DE RENFORTS CIRCULAIRES \\ EQUIDISTANTS ET DE MEME SECTION}

\section{B.1 - On ne tient pas compte de la largeur des renforts.}

Sous l'effet de la pression intérieure il se développe entre chaque renfort et le tuyau une réaction; l'hypothèse envisagée consiste à la considérer comme concentrée sur une circonférence, intersection du tuyau et du plan moyen du renfort. Du fait de l'égalité de section des renforts, de leur équidistance, et de leur nombre infini, le problème est totalement symétrique et la valeur de la réaction est la même à chaque renfort; soit $\mathrm{P}$ sa valeur par unité de longueur circonférentielle.

Nous admettrons qu'on peut confondre le rayon moyen du renfort avec celui du tuyau.
B.1.1. - LES RENFORTS NE SONT PAS PRÉCONTRAINTS, LE TUYAU NE SUBIT PAS D'EFForT LONGITUDINAL.

C'est le cas courant de renforts soudés sur un tuyau libre à ses extrémités.

Calcul de la réaction entre renfort et tuyau.

Le déplacement $W$ d'un point $M$ lié au tuyau sous un renfort peut être considéré comme la somme algébrique de deux déplacements :

$W_{1}$ dû à la pression intérieure seule (tuyau sans renforts);

$\mathrm{W}_{2}$ dû à la réaction du renfort sur le tuyau; $M$ considéré comme lié au renfort subira un déplacement $\mathrm{W} 3$ dû à la réaction du tuyau sur le renfort. 


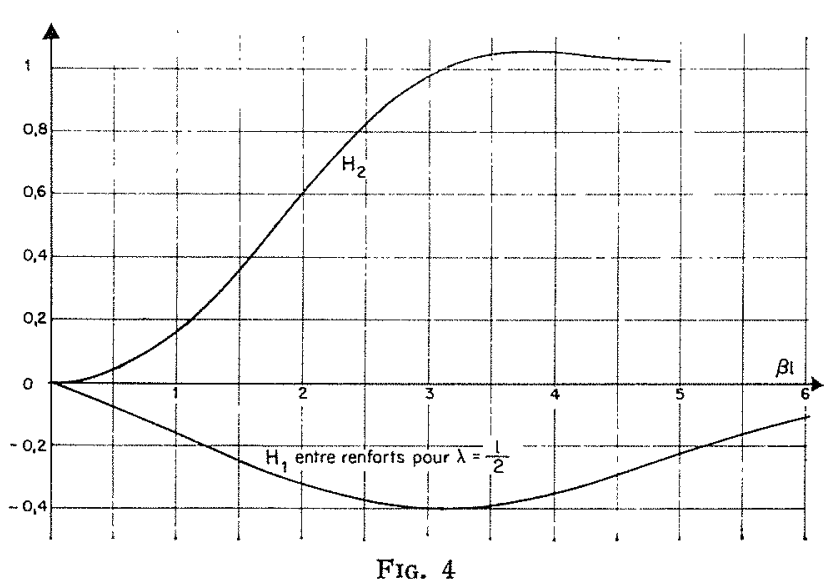

Expression pratique des formules précédentes.

Posons $m=(\mathrm{AK} \beta / 2 e)$. Ce coefficient, sans dimension, sera appelé «coefficient de renforcement du tuyau».

On peut alors écrire :

$$
\left\{\begin{aligned}
\mathrm{P} & =\frac{p \mathrm{~A}}{e(1+m)} \\
\sigma_{c} & =\frac{p \mathrm{R}}{e(1+m)} \\
\sigma_{t} & =\frac{p \mathrm{R}}{e}\left[1-\frac{\mathrm{H} m}{\mathrm{~K}(1+m)}\right] \\
\sigma_{\mathrm{IL}} & =1,816 \frac{p \mathrm{R}}{e} \frac{m_{1}}{\mathrm{~K}(1+m)}
\end{aligned}\right.
$$

sous renfort :

$$
\begin{aligned}
\sigma_{l} & =\sigma_{c} \\
\sigma_{\mathrm{FL}} & =1,816 \frac{p \mathrm{R}}{e} \frac{m \mathrm{H}_{2}}{1+m}
\end{aligned}
$$

ou bien

$$
\sigma_{\mathrm{F} \mathrm{L}_{\mathrm{L}}}=1,816 \mathrm{H}_{2}\left(\frac{p \mathrm{R}}{e}-\sigma_{r}\right)
$$

avec

$$
\mathrm{H}_{2}=\frac{\mathrm{H}_{1}(\beta l)}{\mathrm{K}}=\frac{\operatorname{sh} \beta l-\sin \beta l}{\operatorname{sh} \beta l+\sin \beta l}
$$

Ces formules sont valables dans le cas de pression intérieure $(p>0)$ ou extérieure $(p<0)$.

\section{B.1.2 - LES RENFORTS NE SONT PAS PRÉCONTRAINTS, LE TUYAU SUBIT UN EFFORT LONGITUDINAL.}

L'effort longitudinal appliqué au tuyau peut être dù à la pression (effet de fond, effet Poisson), à la température, au poids, au frottement, etc. Il se traduit par une contrainte longitudinale $\sigma_{t}$ de traction ou de compression.
Nous considérerons que les contraintes du tuyau sous pression résultent de la superposition de deux états :

$1^{\circ}$ Tuyau sans pression soumis à son effort longitudinal;

$2^{\circ}$ Tuyau soumis à la pression sans effort longitudinal.

Ce deuxième état est celui que nous venons d'étudier au paragraphe B.1.1.

En vertu de la loi de Hooke, la contrainte $\sigma_{l}$ engendre un déplacement radial.

$$
\mathrm{W}_{1}=\left(-v \sigma_{l} \mathrm{R} / \mathrm{E}\right)
$$

en l'absence de renforts, $W$ étant positif — c'està-dire tendant à augmenter le diamètre du tuyau - quand $\sigma_{l}$ est une contrainte négative de compression.

Si on applique à la tôle non munie de renfort une pression fictive $p_{f}$ qui ramène son rayon, modifié par l'application de l'effort longitudinal, à celui du renfort, on aura :

$$
p_{f}=\frac{\mathrm{W}_{1} e \mathrm{E}}{\mathrm{R}^{2}}=-\frac{\nu \sigma_{l} e}{\mathrm{R}}
$$

La tôle étant, dans cet état, munie de ses renforts, on fait agir une pression opposée, qui développe entre tuyau et renfort des réactions $\mathrm{P}_{0}$. La superposition de ces deux états rend compte de l'état du tuyau sous pression nulle et sous action de l'effort longitudinal. Les formules du parasraphe B.1.1 peuvent être utilisées.

$\mathrm{P}_{0}, \sigma_{0 c}$ et $\sigma_{0 \mathrm{FI}}$ s'obtiendront en remplaçant $p$ par $p_{f}$.

La contrainte circonférentielle dans la tòle sera la contrainte créée par les forces $\mathrm{P}_{0}$ diminuée de la contrainte qui avait amené la tôle dans son premier état, soit :

$$
\sigma_{0 b}=\frac{p_{f} \mathrm{R}}{e}\left(1-\frac{\mathrm{H} m}{\mathrm{~K}(1+m)}\right)+v \sigma_{l}=\frac{v \sigma_{l} \mathrm{H} m}{\mathrm{~K}(1+m)}
$$

Si on superpose à cet état l'état de pression intérieure, nous aurons finalement :

$$
\left\{\begin{aligned}
\mathrm{P} & =\frac{-v \sigma_{l} \mathrm{~A}}{\mathrm{R}(1+m)}+\frac{p \mathrm{~A}}{e(1+m)} \\
\sigma_{c} & =\frac{-v \sigma_{l}}{1+m}+\frac{p \mathrm{R}}{e(1+m)} \\
\sigma_{t} & =\frac{v \sigma_{l} \mathrm{Hm}}{\mathrm{K}(1+m)}+\frac{p \mathrm{R}}{e}\left(1-\frac{\mathrm{H} m}{\mathrm{~K}(1+m)}\right) \\
\sigma_{\mathrm{FL}} & =1,816 \frac{m \mathrm{H}_{1}}{\mathrm{~K}(1+m)}\left(\frac{p \mathrm{R}}{e}-v \sigma_{l}\right)
\end{aligned}\right.
$$


sous renfort :

$$
\left.\begin{array}{rl}
\sigma_{t} & =\frac{v \sigma_{l} m}{1+m}+\frac{p \mathbf{R}}{e(1+m)} \neq \sigma_{c} \\
\sigma_{\mathrm{FL}} & =1,816 \frac{m H_{2}}{1+m}\left(\frac{p \mathrm{R}}{e}-{ }^{v} \sigma_{l}\right)
\end{array}\right\}
$$

NotA. - $P_{0}=-v \sigma_{l} \mathrm{~A} /[\mathrm{R}(1+m)]$ négatif signifie que cette force tend à séparer le renfort de la tôle, alors que $P_{0}$ positif tend à plaquer la tôle sur le renfort, ceci dans le cas courant où le renfort est extérieur au tuyau.

Calcul de la contrainte $\sigma_{l}$ dans les cas usuels.

La contrainte $\sigma_{l}$ est ordinairement le quotient de l'effort longitudinal appliqué par la section de métal du tuyau. Il est donc essentiel de bien. définir cet effort longitudinal; si c'est chose facile, dans le cas d'un effet de fond, d'un effort de frottement, d'un effort thermique (à condition qu'il y ait uniformité de température entre tôle et renforts), il nous a par contre paru nécessaire d'en préciser le calcul dans le cas très courant d'un tuyau à extrémités encastrées soumis longitudinalement à l'effet Poisson de la pression intérieure.

On calculera les déplacements du tuyau supposé libre en superposant deux états :

1 Pression seule agissante, sans action des renforts;

2 Renforts seuls agissants, sans pression.

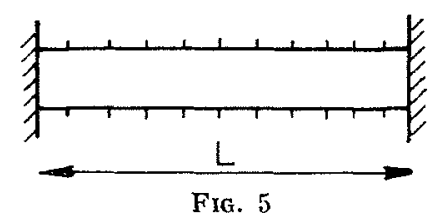

Allongement dîu $\dot{a}$ la pression seule :

$$
-\frac{\nu}{\mathrm{E}} \frac{p \mathrm{R}}{e} \mathrm{~L}
$$

Allongement dû aux réactions des renforts :

Nous sommes bien obligés de faire ici l'hypothèse d'un nombre fini de renforts, répartis sur la longueur I du tuyau; leur nombre sera supposé suffisamment grand pour qu'on admette l'égalité de toutes les réactions entre renfort et tuyau. Dans ces conditions, calculons l'allongement produit par $n$ forces $\mathrm{P}$ équidistantes sur la portion de tuyau comprise entre deux forces : en un point d'abscisse $\lambda(0<\lambda<l)$, la flèche

$$
\mathrm{W}=\frac{\mathrm{PR}^{2} \beta}{2 \mathrm{eE}} \mathrm{H}(\beta \lambda)
$$

engendre une contrainte circonférentielle

$$
\sigma=(\mathrm{E} w / \mathrm{R})
$$

qui appliquée sur l'élément $d \lambda$ situé à l'abscisse $\lambda$ augmente sa longueur de :

$$
\frac{\nu \sigma d \lambda}{\mathrm{E}}=\frac{\nu \mathrm{PR} \beta \mathrm{H}}{2 e \mathrm{E}} d \lambda
$$

soit pour la portion $l$ du tuyau :

$$
2 \int_{0}^{1 / 2} \frac{\nu \mathrm{PR} \beta \mathrm{H}(\beta \lambda)}{2 e \mathrm{E}} d \lambda=\frac{\nu \mathrm{PR}}{e \mathrm{E}}
$$

Pour la totalité du tuyau, l'allongement sera :

$$
\frac{n \cup \mathrm{P} R}{e \mathrm{E}}
$$

Allongement dî̀ $\dot{a}$ la superposition des deux états :

$\Delta \mathrm{L}=-\frac{v p \mathrm{RL}}{\mathrm{E} e}+\frac{n v \mathrm{PR}}{e \mathrm{E}}=\frac{v p \mathrm{RL}}{e \mathrm{E}}\left(\frac{1}{(e l / \mathrm{A})(1+m)}-1\right)$

d'oủ la contrainte nécessaire pour créer un allongement de sens contraire :

$$
\mid \sigma_{l}=-\frac{\mathrm{E} \Delta \mathrm{L}}{\mathrm{L}}=\frac{v p \mathrm{R}}{e}\left[1-\frac{1}{(e l / \mathrm{A})(1+m)}\right]
$$

P.1.3 - LES RENFORTS SONT PRECONTHMNTS, LE TUYAU NE SUBIT PAS D'EFFORT IONGITUDINAL.

Supposons pour l'instant que la précontrainte soit réalisée par emmanchement à chaud des renforts de rayon initial intérieur $\mathrm{R} c$, inférieur au rayon initial extérieur $\mathrm{R} t$ du tuyau :

$$
\mathrm{R} t-\mathrm{R} c=\Delta \mathrm{R} \text {. }
$$

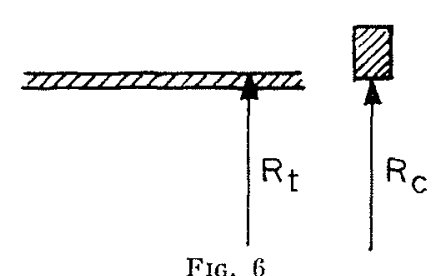

Nous raisonnerons comue au paragraphe. 3.1.2. Si on applique à la tôle non munie de renforts une pression extérieure fictive $p_{f}$ qui ramène son rayon à celui du renfort, on aura : $p_{f}=\left(e \mathrm{~F} \Delta \mathrm{R} / \mathrm{R}^{2}\right)$. La tôle étant, dans cet étal. munie de ses renforts, on fait agir une pression opposée, intérieure cette fois, gui développe entre tuyau et renfort des réactions $P_{0}$. La superposition de ces deux śtats crée l'état de précontrainte du tuyau sous pression nulle. Dès lors, les formules du paragraphe B.1.-1 s'appliquent intégralement :

$\mathrm{P}_{0}, \sigma_{0,}$ et $\sigma_{0 \mathrm{~F}}$, s'obtiendront en remplacinl $p$ par $p_{f}$. 
La contrainte circonférentielle dans la tôle sera la contrainte créée par les forces $P_{0}$ diminuée de la contrainte de compression $(E \Delta R / R)$ qui avait amené la tôle dans son premier état, soit :

$\sigma_{0 t}=\frac{p_{f} \mathrm{R}}{\boldsymbol{e}}\left(1-\frac{\mathrm{Hm}}{\mathrm{K}(1+m)}\right)-\frac{\mathrm{E} \Delta \mathrm{R}}{\mathrm{R}}=-\frac{\mathrm{E} \Delta \mathrm{RH} m}{\mathrm{RK}(1+m)}$

Si on superpose à cet état l'état de pression intérieure, nous aboutissons finalement aux formules :

$$
\left\{\begin{aligned}
\mathrm{P} & =\frac{\mathrm{EA} \Delta \mathrm{R}}{\mathrm{R}^{2}(1+m)}+\frac{p \mathrm{~A}}{e(1+m)} \\
\sigma_{c} & =\frac{\mathrm{E} \Delta \mathrm{R}}{\mathrm{R}(1+m)}+\frac{p \mathrm{R}}{e(1+m)} \\
\sigma_{t} & =\frac{\mathrm{E} \Delta \mathrm{RH} m}{\mathrm{RK}(1+m)}+\frac{p \mathrm{R}}{e}\left(1-\frac{\mathrm{Hm}}{\mathrm{K}(1+m)}\right) \\
\sigma_{\mathrm{FL}} & =1,816^{1} \frac{m \mathrm{H}_{1}}{\mathrm{~K}(1+m)}\left(\frac{\mathrm{E} \Delta \mathrm{R}}{\mathrm{R}}+\frac{p \mathrm{R}}{e}\right)
\end{aligned}\right.
$$

sous renfort :

$$
\left.\begin{array}{rl}
\sigma_{t} & =-\frac{\mathrm{E} \Delta \mathrm{Rm}}{\mathrm{R}(1+m)}+\frac{p \mathrm{R}}{e(1+m)} \neq \sigma_{c} \\
\sigma_{\mathrm{FL}} & =1,816 \frac{m \mathrm{H}_{2}}{1+m}\left(\frac{\mathrm{E} \Delta \mathrm{R}}{\mathrm{R}}+\frac{p \mathrm{R}}{e}\right)
\end{array}\right\}
$$

\section{B.1.4-LES RENFORTS SONT PRÉCONTRAINTS, LE} TUYAU SUBIT UN EFFORT LONGITUDINAL.

Les efforts de précontrainte (indice 0) se superposeront et on aboutira aux expressions suivantes :

$$
\left\{\begin{aligned}
\mathrm{P} & =\left(\frac{\mathrm{E} \Delta \mathrm{R}}{\mathrm{R}}-v \sigma_{l} \frac{\mathrm{A}}{\mathrm{R}(1+m)}+\frac{p \mathrm{~A}}{e(1+m)}\right. \\
\sigma_{c} & =\left(\frac{\mathrm{E} \Delta \mathrm{R}}{\mathrm{R}}-v \sigma_{l}\right) \frac{1}{1+m}+\frac{p \mathrm{R}}{e(1+m)} \\
\sigma_{t} & =\left(-\frac{\mathrm{E} \Delta \mathrm{R}}{\mathrm{R}}+v \sigma_{l}\right) \frac{\mathrm{H} m}{\mathrm{~K}(1+m)} \\
& \quad-\frac{p \mathrm{R}}{e}\left(1-\frac{\mathrm{Hm}}{\mathrm{K}(1+m)}\right) \\
\sigma_{\mathrm{FL}} & =1,816 \mid \frac{m \mathrm{H}_{1}}{\mathrm{~K}(1+m)}\left(\frac{\mathrm{E} \Delta \mathrm{R}}{\mathrm{R}}-v \sigma_{l}+\frac{p \mathrm{R}}{e}\right)
\end{aligned}\right.
$$

sous renfort :

$$
\left.\begin{array}{rl}
\sigma_{t} & =\left(-\frac{\mathrm{E} \Delta \mathrm{R}}{\mathrm{R}}+\nu \sigma_{l}\right) \frac{m}{1+m}+\frac{p \mathrm{R}}{e(1+m)} \\
\sigma_{\mathrm{FL}} & =1,816 \frac{m \mathrm{H}_{2}}{1+m}\left(\frac{\mathrm{E} \Delta \mathrm{R}}{\mathrm{R}}-\nu \sigma_{l}+\frac{p \mathrm{R}}{e}\right)
\end{array}\right\}
$$

Nota. - Ces formules supposent que l'opération de précontrainte relative à $\Delta R$ a été effectuée sur un tuyau à extrémités libres.

\section{B.2 - On tient compte de la largeur des ren- forts.}

B.2.1. - HYPothèse D'UNE PRESSION UNIFORMé-

Cette pression $q$ entre renfort et tuyau règne sur la largeur de contact $a$ du renfort. Ce cylindre de pression se reproduit à chaque pas $l$, espacement des renforts d'axe en axe.

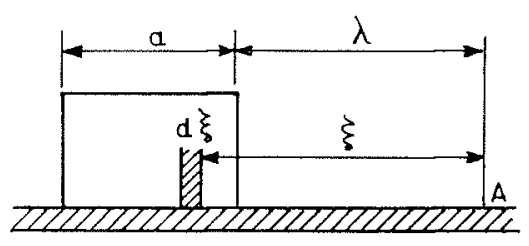

FIG. 7

Par un raisonnement analogue à celui du paragraphe A-1, on considère qu'un anneau $d \xi$ de charge $q d \xi$ par unité de longueur circonférentielle produit en A une flèche $d W$

$$
d \mathrm{~W}=\frac{q d \xi \mathrm{R}^{2} \beta}{2 e \mathrm{E}} e^{-\beta \xi}(\sin \beta \xi+\cos \beta \xi)
$$

Le même processus de calcul nous conduit aux expressions suivantes, dans le cas d'un nombre infini de renforts :

$\mathrm{W}$ sous le renfort $=\frac{q \mathrm{R}^{2}}{2 e \mathrm{E}} \mathrm{K}^{\prime}(\beta l)$

$\mathrm{W}$ entre renforts $=\frac{q \mathrm{R}^{2}}{2 e \mathrm{E}} \mathrm{H}^{\prime}(\beta \lambda)$ 
expressions comparables aux expressions [3] et [4] du paragraphe A-3 mais avec :

$$
\begin{aligned}
\mathrm{K}^{\prime}(\beta l) & =\frac{\operatorname{ch} \beta l-\cos \beta l+\operatorname{ch} \beta a \cos \beta(a-l)-\operatorname{ch} \beta(a-l) \cos \beta a}{\operatorname{ch} \beta l-\cos \beta l} \\
\mathrm{H}^{\prime}(\beta \lambda) & =[\operatorname{ch} \beta(l-\lambda) \cos \beta \lambda-\operatorname{ch} \beta \lambda \cos \beta(l-\lambda)+\operatorname{ch} \beta(a+\lambda) \cos \beta(a+\lambda-l) \\
& -\operatorname{ch} \beta(a+\lambda-l) \cos \beta(a+\lambda)] \frac{1}{\operatorname{ch} \beta l-\cos \beta l}
\end{aligned}
$$

On vérifie que : $\mathrm{H}^{\prime}=\mathrm{K}^{\prime}$ si $\lambda=0$ ou si $\lambda=l-a$, c'est-à-dire au bord du renfort.

Les formules du paragraphe B-1-1 deviennent alors :

$$
\left\{\begin{aligned}
q & =\frac{p \mathrm{~A}}{a e\left(1+m^{\prime}\right)} \quad \text { avec } m^{\prime}=-\frac{\mathrm{AK}^{\prime}}{2 a e} \\
\sigma_{i} & =\frac{p \mathrm{R}}{e\left(1+m^{\prime}\right)} \\
\sigma_{t} & =\frac{p \mathrm{R}}{e}\left[1-\frac{\mathrm{H}^{\prime} m^{\prime}}{\mathrm{K}^{\prime}\left(1+m^{\prime}\right)}\right] \\
\sigma_{\mathrm{FL}} & =1,816 \frac{p \mathrm{R}}{e} \cdot \frac{\mathrm{H}^{\prime}{ }_{1} m^{\prime}}{\mathrm{K}^{\prime}\left(1+m^{\prime}\right)}
\end{aligned}\right.
$$

avec $H^{\prime}{ }_{1}=\frac{\operatorname{sh} \beta \lambda \sin \beta(l-\lambda)-\operatorname{sh} \beta(l-\lambda) \sin \beta \lambda+\operatorname{sh} \beta(a+\lambda) \sin \beta(a+\lambda-l)-\operatorname{sh} \beta(a+\lambda-l) \sin \beta(\alpha+\lambda)}{\operatorname{ch} \beta l-\cos \beta l}$

En réalité, la répartition réelle de pression sous un renfort large est un problème très complexe. L'hypothèse envisagée ici n'est qu'une approximation qu'on peut admettre dans le cas d'un renfort suffisamment large et mince, capable de subir une légère déformation longitudinale. Aussi ne la mentionnons-nous que d'un point de vue théorique, sans l'utiliser par la suite dans les applications courantes.

B.2.2. - CAS USUEL DES RENFORTS EN PROFILÉS.

Chaque renfort peut être formé d'un fer U, d'un groupe de deux cornières ou de deux plats.

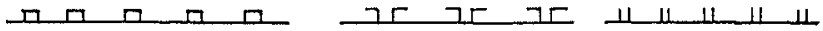

FIG. $8 a$

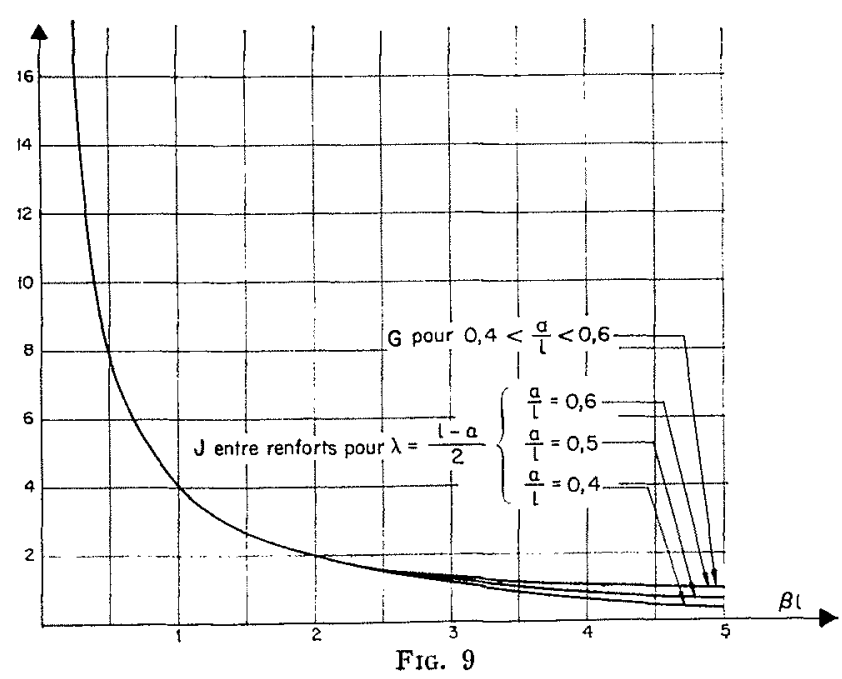

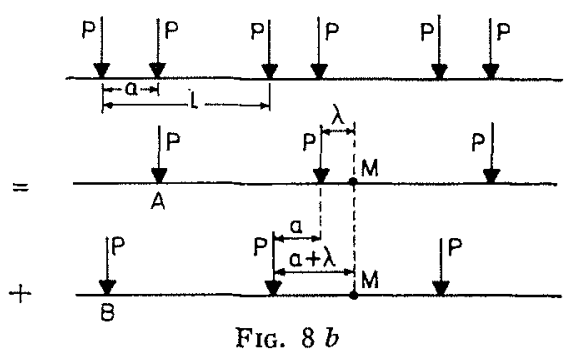

Le problème se ramène à l'étude d'un double système de forces $\mathrm{P}$, de pas $l$, décalées d'une distance $a$.

Nous superposerons donc le déplacement $W_{1}$ créé en un point $M$ d'abscisse $\lambda$ par le premier système de forces $\mathrm{P}$, soit

$W_{1}=\frac{P R^{2} \beta}{2 e E} H(\beta \lambda)$ (d'après les expressions [3] et [4] du paragraphe A-3), au déplacement $W_{2}$ créé au même point par le deuxième système de forces, soit

$$
\mathrm{W}_{2}=\frac{\mathrm{PR}^{2} \beta}{2 e \mathrm{E}} \mathrm{H}[\beta(\lambda+a)] .
$$


Nous pouvons done écrire :

$$
\begin{aligned}
& W \text { en } A \text { ou en } B=\frac{P R^{2} \beta}{2 e E} G, \\
& W \text { entre renforts }=\frac{P R^{2} \beta}{2 e E} J
\end{aligned}
$$

avec $\mathrm{G}=\mathrm{K}+\frac{\operatorname{sh} \beta a \cos \beta(l-a)+\operatorname{ch} \beta a \sin \beta(l-a)+\operatorname{sh} \beta(l-a) \cos \beta a+\operatorname{ch} \beta(l-a) \sin \beta a}{\operatorname{ch} \beta l-\cos \beta l}$

et $\mathrm{J}=\mathrm{H}[\beta \lambda]+\mathrm{H}[\beta(\lambda+a)]$ (H donné par l'expression [4] du paragraphe $\mathrm{A}-3$ ).

Par un raisonnement analogue à celui du paragraphe B-1-1, nous obtiendrons les formules suivantes, dans le cas le plus général de renforts précontraints et d'un effort longitudinal agissant sur le tuyau :

$$
\begin{gathered}
\text { en posant } m_{1}=\frac{\mathrm{AG} \beta}{4 e}: \\
\mathrm{P}=\left[\frac{\mathrm{E} \Delta \mathrm{R}}{\mathrm{R}}-v \sigma_{l}\right] \frac{\mathrm{A}}{2 \mathrm{R}\left(1+m_{1}\right)}+\frac{p \mathrm{~A}}{2 e\left(1+m_{1}\right)} \\
\sigma_{c}=\left[\frac{\mathrm{E} \Delta \mathrm{R}}{\mathrm{R}}-v \sigma_{l}\right] \frac{1}{1+m_{1}}+\frac{p \mathrm{R}}{e\left(1+m_{1}\right)} \\
\sigma_{t}=\left[-\frac{\mathrm{E} \Delta \mathrm{R}}{\mathrm{R}}+v \sigma_{l}\right\rfloor \frac{\mathrm{J} m_{1}}{\mathrm{G}\left(1+m_{1}\right)}+\frac{p \mathrm{R}}{e}\left(1-\frac{\mathrm{J} m_{1}}{\mathrm{G}\left(1+m_{1}\right)}\right) \\
\sigma_{\mathrm{FL}}=1,816 \frac{m_{1} \mathrm{~J}_{1}}{\mathrm{G}\left(1+m_{1}\right)}\left[\frac{\mathrm{E} \Delta \mathrm{R}}{\mathrm{R}}-v \sigma_{l}+\frac{p \mathrm{R}}{e}\right]
\end{gathered}
$$

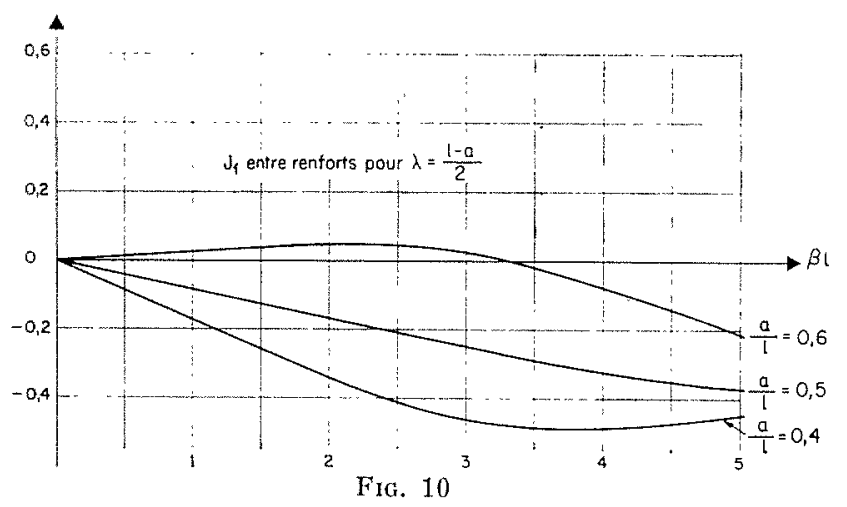

au bord d'un renfort:

$$
\left.\begin{array}{rl}
\sigma_{t} & =\left[-\frac{\mathrm{E} \Delta \mathrm{R}}{\mathrm{R}}+v \sigma_{l}\right] \frac{m_{1}}{1+m_{1}}+\frac{p \mathrm{R}}{e\left(1+m_{1}\right)} \\
\sigma_{\mathrm{FL}} & =1,816 \frac{m_{1} \mathrm{~J}_{2}}{1+m_{1}}\left[\frac{\mathrm{E} \Delta \mathrm{R}}{\mathrm{R}}-v \sigma_{l}+\frac{p \mathrm{R}}{e}\right]
\end{array}\right\}
$$

avec: $\mathrm{J}_{1}=\mathrm{H}_{1}[\beta \lambda]+\mathrm{H}_{1}[\beta(\lambda+a)]\left(\mathrm{H}_{1}\right.$ explicité dans le paragraphe B-1-1). 


$$
\begin{aligned}
J_{2} & =\frac{J_{1}(\beta l)}{G} \\
& =\frac{\operatorname{sh} \beta l-\sin \beta l+\operatorname{sh} \beta a \cos \beta(l-a)+\operatorname{sh} \beta(l-a) \cos \beta a-\operatorname{ch} \beta a \sin \beta(l-a)-\operatorname{ch} \beta(l-a) \sin \beta a}{\operatorname{sh} \beta l+\sin \beta l+\operatorname{sh} \beta \alpha \cos \beta(l-a)+\operatorname{sh} \beta(l-a) \cos \beta a+\operatorname{ch} \beta a \sin \beta(l-a)+\operatorname{ch} \beta(l-a) \sin \beta a}
\end{aligned}
$$

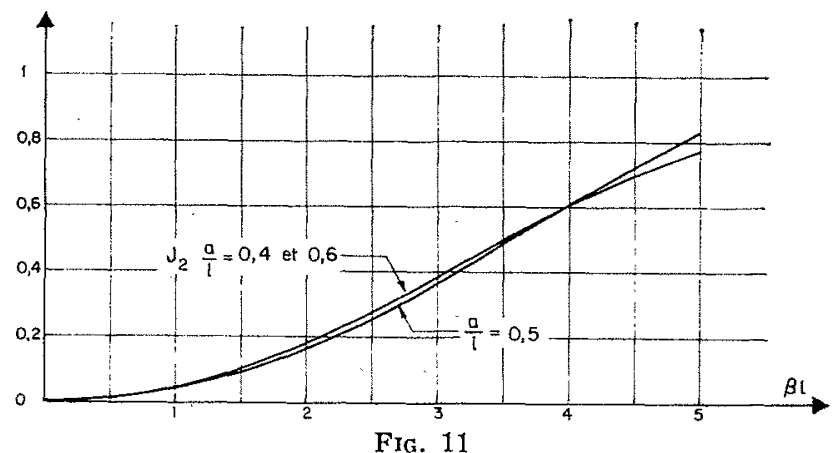

Il est bien entendu que A représente, dans les schémas de renforts en profilés indiqués plus haut, la section du fer $U$, ou bien la section de l'ensemble des deux cornières ou des deux plats. P représente la réaction par unité de longueur circonférencielle, à l'un des bords du renfort.

Nous pourrons calculer de la même manière la contrainte longitudinale due à l'effet Poisson de la pression intérieure, soit :

$$
\sigma_{l}=\frac{\nu p \mathrm{R}}{e}\left[1-\frac{Z}{(e l / \mathrm{A})\left(1+m_{1}\right)}\right] \text { avec } \mathrm{Z}=\frac{1+\operatorname{ch} \beta a \cos \beta a}{2}
$$

\section{C. - CAS D'UN RENFORT ISOLE}

\section{1 - On ne tient pas compte de la largeur du renfort.}

\section{C.1.1. - RENFort NON PRÉCONTRAINT, SANS EFFORT I.ONGITUDINAL.}

Dire que le renfort est isolé revient à considérer que la distance $l$ entre deux renforts devient infinie. Pour $l$ infini, les expressions $\mathrm{K}, \mathrm{H}, \mathrm{H}_{1}$ et $\mathrm{H}_{2}$ du paragraphe B-1 se transforment comme suit :

$\mathrm{K}$ tend vers $\mathbf{1}$;

$H(\beta \lambda)$ tend vers $\varphi(\beta \lambda)=e^{-\beta \lambda}(\sin \beta \lambda+\cos \beta \lambda)$;

$\mathrm{H}_{1}(\beta \lambda)$ tend vers $\psi(\beta \lambda)=e^{-\beta \lambda}(\cos \beta \lambda-\sin \beta \lambda)$;

$\mathrm{H}_{2}$ lend vers 1 ;
Posons $\mu=\mathrm{A} \beta / 2 e$. Les formules du paragraphe B-1-1 deviennent :

$$
\begin{aligned}
\mathrm{P} & =\frac{p \mathrm{~A}}{e(1+\mu)} \\
\sigma_{e} & =\frac{p \mathrm{R}}{e(1+\mu)} \\
\sigma_{t} & =\frac{p \mathrm{R}}{e}\left(1-\frac{\mu^{\mu}}{1+\mu}\right) \\
\sigma_{\mathrm{FL}} & =1,816 \frac{p \mathrm{R}}{e} \frac{\mu 4}{1+\mu}
\end{aligned}
$$

sous renfort:

$$
\left.\begin{array}{l}
\sigma_{t}=\sigma_{c} \\
\sigma_{\mathrm{FL}}=1,816 \frac{p \mathrm{R}}{e} \frac{\mu}{1+! \cdot} \\
\quad \text { ou bien } 1,816\left(\frac{p \mathrm{R}}{e}-\sigma_{c}\right)
\end{array}\right\}
$$


On peut aussi introduire la notion de « largeur associée $l_{\mathrm{A}}$ en considérant que la contrainte de pression $\sigma_{c}$ ou $\sigma_{t}$ sous renfort est la même que celle produite dans un anneau cylindrique de largeur $l_{\mathrm{A}}=(2 / \beta)=1,556 \sqrt{\mathrm{R} e}$ et d'épaisseur $e+e_{\mathrm{A}}, e_{\mathrm{A}}$ étant l'épaisseur répartie du renfort sur la largeur $l_{\mathrm{A}}$ soit $e_{\mathrm{A}}=\left(\mathrm{A} / l_{\mathrm{A}}\right)$. Cette largeur associée n'a plus de sens dans le cas d'un renfort précontraint ou d'un tuyau soumis à un effort longitudinal.

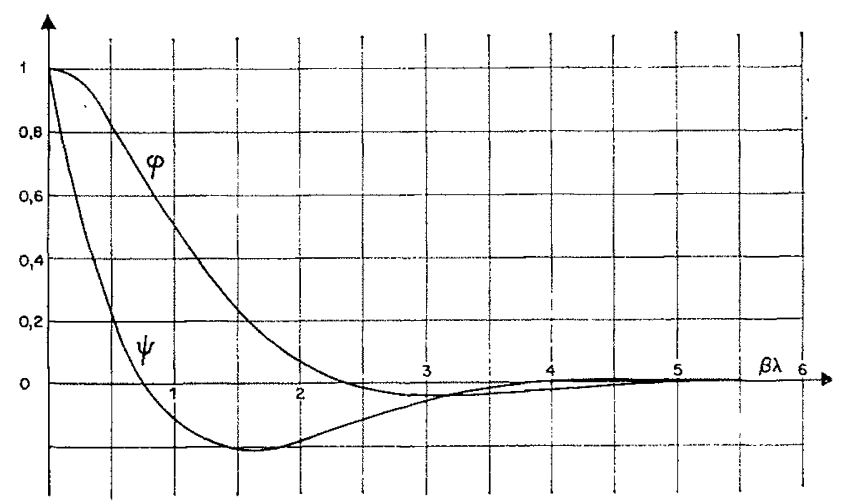

FIG. 12

\section{C.1.2. - RENFORT PRÉCONTRAINT, TUYAU SOUMIS A} UN EFFOR'T LONGITUDINAL.

Les formules du paragraphe B-1-4 se transforment de la même manière dans les formules générales suivantes :

$$
\left\{\begin{aligned}
& \mathrm{P}=\left(\frac{\mathrm{E} \Delta \mathrm{R}}{\mathrm{R}}-v \sigma_{l}\right) \frac{\mathrm{A}}{\mathrm{R}(1+\mu)}+\frac{p \mathrm{~A}}{e(1+\mu .)} \\
& \sigma_{c}=\left(\frac{\mathrm{E} \Delta \mathrm{R}}{\mathrm{R}}-v \sigma_{l}\right) \frac{1}{1+\mu}+\frac{p \mathrm{R}}{e(1+\mu)} \\
& \sigma_{t}=\left(-\frac{\mathrm{E} \Delta \mathbf{R}}{\mathrm{R}}+v \sigma_{l}\right) \frac{\varphi \mu}{1+\mu} \\
& \quad+\frac{p \mathrm{R}}{e}\left(1-\frac{\varphi \mu}{1+\mu}\right) \\
& \sigma_{\mathrm{FL}}=1,816 \frac{\psi \mu}{1+\mu}\left(\frac{\mathrm{E} \Delta \mathbf{R}}{\mathbf{R}}-v \sigma_{t}+\frac{p \mathbf{R}}{e}\right)
\end{aligned}\right.
$$

sous renfort :

$$
\left.\begin{array}{l}
\sigma_{t}=\left(-\frac{\mathrm{E} \Delta \mathrm{R}}{\mathrm{R}}+v \sigma_{l}\right) \frac{\mu}{1+\mu}+\frac{p \mathrm{R}}{e(1+\mu .)} \\
\sigma_{\mathrm{FL}}=1,816 \frac{\mu}{1+\mu}\left(\frac{\mathrm{E} \Delta \mathrm{R}}{\mathrm{R}}-v \sigma_{l}+\frac{p \mathrm{R}}{e}\right)
\end{array}\right\}
$$

La contrainte longitudinale due à l'effet Poisson de la pression intérieure sera :

$$
\sigma_{l}=\frac{v p \mathrm{R}}{e}
$$

\section{C.2 - On tient compte de la largeur du renfort.}

\section{C.2.1 - Renfort non précontraint, SANS EFfort} LONGITUDINAL.

Pour $l$ infini, les expressions $G, J, J_{1}, J_{2}$, du paragraphe B-2-2 se transforment comme suit :

$\mathrm{G}$ tend vers $1+\varphi(\beta a) \quad=\mathrm{G}^{\prime}$

$\mathrm{J}$ tend vers $\varphi[\beta \lambda]+\varphi[\beta(\lambda+\alpha)]=\mathrm{J}^{\prime}$

$\mathrm{J}_{1}$ tend vers $\psi[\beta \lambda]+\psi[\beta(\lambda+a)]=\mathrm{J}_{1}^{\prime}$

$\mathrm{J}_{2}$ tend vers $\frac{1+\psi(\beta a)}{1+0(\beta a)}=\mathrm{J}^{\prime}$

D'où les expressions :

en posant $m_{1}^{\prime}=\left(\mathrm{AG}^{\prime} \beta / 4 e\right)$ :

$$
\begin{aligned}
\mathrm{P} & =\frac{p \mathrm{~A}}{2 e\left(1+m_{1}^{\prime}\right)} \\
\sigma_{c} & =\frac{p \mathrm{R}}{e\left(1+m_{1}^{\prime}\right)} \\
\sigma_{t} & =\frac{p \mathrm{R}}{e}\left(1-\frac{\mathrm{J}^{\prime} m_{1}^{\prime}}{\mathrm{G}^{\prime}\left(1+m_{1}\right)}\right) \\
\sigma_{\mathrm{FL}} & =1,816 \frac{p \mathrm{R}}{e} \frac{m_{1}^{\prime} \mathrm{J}_{1}^{\prime}}{\mathrm{G}^{\prime}\left(1+m_{1}^{\prime}\right)}
\end{aligned}
$$

au bord du renfort :

$$
\left.\begin{array}{rl}
\sigma_{t} & =\sigma_{c} \\
\sigma_{\mathrm{FL}} & =1,816 \frac{p \mathrm{R}}{e} \frac{m_{1}^{\prime} \mathrm{J}_{2}^{\prime}}{1+m_{1}^{\prime}}
\end{array}\right\}
$$
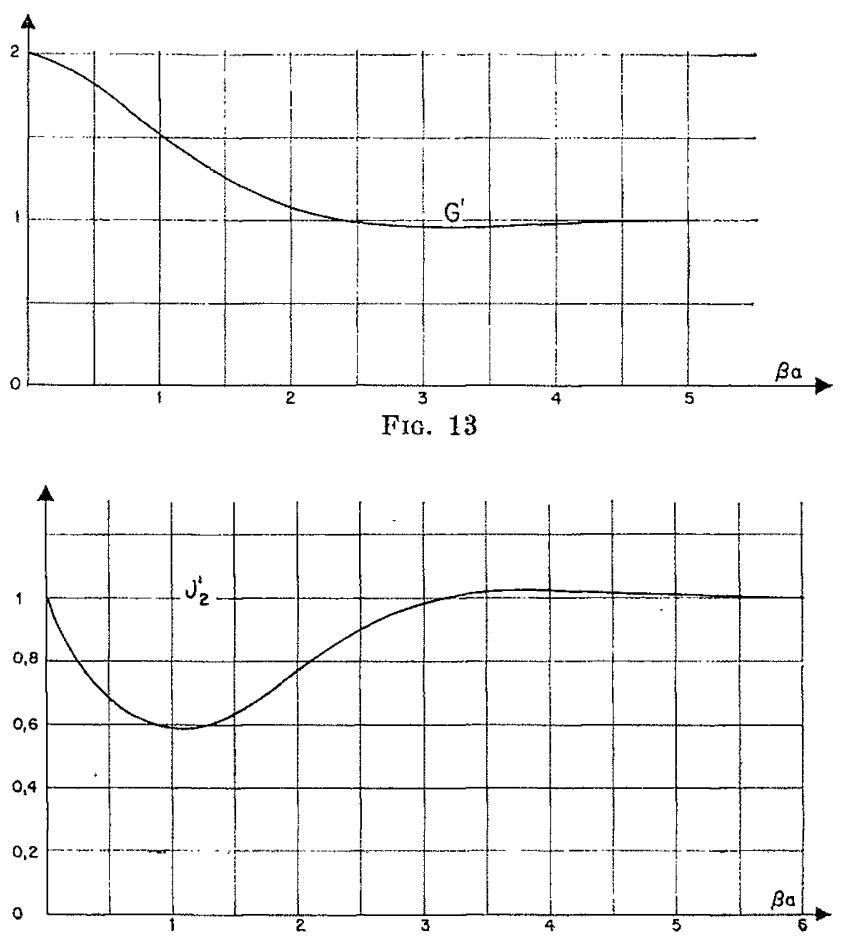

Fig. 14 


\section{C.2.2. - RENFORT PRÉCONTRAINT, TUYAU SOUMIS A UN EFFORT LONGITUDINAL.}

Nous obtiendrons pareillement:

$$
\begin{aligned}
& \mathrm{P}=\left(\frac{\mathrm{E} \Delta \mathrm{R}}{\mathrm{R}}-v \epsilon_{l}\right) \frac{\mathrm{A}}{2 \mathrm{R}\left(1+m_{1}^{\prime}\right)}+\frac{p \mathrm{~A}}{2 e\left(1+m^{\prime}{ }_{1}\right)} \\
& \sigma_{c}=\left(\frac{\mathrm{E} \Delta \mathrm{R}}{\mathrm{R}}-v \sigma_{l}\right) \frac{1}{1+m_{1}^{\prime}}+\frac{p \mathrm{R}}{e\left(1+m_{1}^{\prime}\right)} \\
& \sigma_{t}=\left(-\frac{\mathrm{E} \Delta \mathrm{R}}{\mathrm{R}}+v_{l}\right) \frac{\mathrm{J}^{\prime} m_{1}^{\prime}}{\mathrm{G}^{\prime}\left(1+m_{1}^{\prime}\right)} \\
&+\frac{p \mathrm{R}}{e}\left(1-\frac{\mathrm{J}^{\prime} m_{1}^{\prime}}{\mathrm{G}^{\prime}\left(1+m_{1}^{\prime}\right)}\right) \\
& \sigma_{\mathrm{J}, \mathrm{F}}=1,816 \frac{m_{1}^{\prime} \mathrm{J}_{1}^{\prime}}{\mathrm{G}^{\prime}\left(1+m_{1}^{\prime}\right)}\left[\frac{\mathrm{E} \Delta \mathrm{R}}{\mathrm{R}}-\nu \sigma_{l}+\frac{p \mathrm{R}}{e}\right]
\end{aligned}
$$

au bord d'un renfort :

$$
\left\{\begin{aligned}
\sigma_{t} & =\left(-\frac{\mathrm{E} \Delta \mathrm{R}}{\mathrm{R}}+v \sigma_{l}\right) \frac{m_{1}^{\prime}}{1+m_{1}^{\prime}}+\frac{p \mathrm{R}}{e\left(1+m_{1}^{\prime}\right)} \\
\sigma_{\mathrm{FL} L} & =1,816 \frac{m_{1}^{\prime} \mathrm{J}_{2}^{\prime}}{1+m_{1}^{\prime}}\left[\frac{\mathrm{E} \Delta \mathrm{R}}{\mathrm{R}}-v \sigma_{l}+\frac{p \mathrm{R}}{e}\right]
\end{aligned}\right.
$$

La contrainte longitudinale due à l'effet Poisson de la pression intérieure sera:

$$
\sigma_{l}=\frac{\vartheta p \mathrm{R}}{e}
$$

\section{C.3. - Exemple relatif aux renforts isolés.}

A titre d'exemple, et pour montrer la différence que donnent les deux méthodes de calcul, nous étudierons le comportement à la pression intérieure d'un tuyau ancré à ses deux extrémités, de diamètre $2 \mathrm{~m}$, d'épaisseur $16 \mathrm{~mm}$ muni d'un renfort formé de deux cornières $80 \times 40$ $\times 6$ espacées de $70 \mathrm{~mm}$. Pression intérieure $22,4 \mathrm{~kg} / \mathrm{cm}^{2}$.

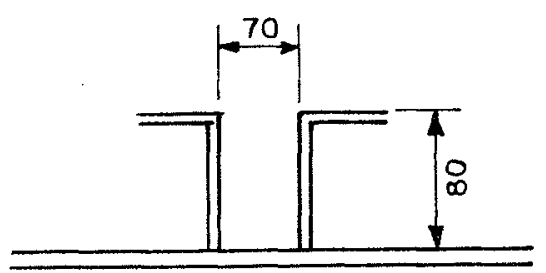

Fig. 15

Section totale de renfort : $A=1376 \mathrm{~mm}^{2}$.

$$
\beta=\frac{1,285}{\sqrt{1000 \times 16}}=0,01015
$$

$1^{\circ}$ D'après C.1.2 (on considère l'effet du renfort comme concentré sur un seul cercle) :

$$
\begin{aligned}
\mu & =0,435 ; \\
\sigma_{l} & =4,2 \mathrm{~kg} / \mathrm{mm}^{2} \text { dù à l'effet Poisson; } \\
\sigma_{c} & =8,87 \mathrm{~kg} / \mathrm{mm}^{2} ; \\
\sigma_{t} & =10,13 \mathrm{~kg} / \mathrm{mm}^{2} \text { sous renfort; } \\
\sigma_{\mathrm{FL}} & =7 \mathrm{~kg} / \mathrm{mm}^{2} \text { sous renfort. }
\end{aligned}
$$

En tenant compte de l'effet Poisson circonférentiel des contraintes de flexion, nous aurons : $\sigma_{t}$ total $=10,13+0,3 \times \sigma_{\mathrm{FL}}=12,23 \mathrm{~kg} / \mathrm{mm}^{2}$ sur fibre intérieure;

$\sigma_{t}$ total $=10,13-0,3 \times \sigma_{\mathrm{FL}}=8,03 \mathrm{~kg} / \mathrm{mm}^{2}$ sur fibre extérieure.

$2^{\circ}$ D'après C-2-2 (on tient compte de l'espacement des deux cornières) :

$$
\begin{aligned}
a & =70 ; \\
\beta a & =0,71 ; \\
\mathrm{G}^{\prime} & =1,69 \\
\mathrm{~J}_{2}^{\prime} & =0,65 \\
m_{1}^{\prime} & =0,368 ; \\
\sigma_{c} & =9,32 \mathrm{~kg} / \mathrm{mm}^{2} ; \\
\sigma_{t} & =10,58 \mathrm{~kg} / \mathrm{mm}^{2} \text { au bord du renfors } ; \\
\sigma_{\mathrm{FL}} & =4,05 \mathrm{~kg} / \mathrm{mm}^{2} \text { au bord du renfort. }
\end{aligned}
$$

En tenant compte de l'effet Poisson circonférentiel des contraintes de flexion :

$$
\begin{aligned}
\sigma_{t} \text { total }= & 10,58+0,3 \sigma_{\mathrm{FL}}=11,79 \mathrm{~kg} / \mathrm{mm}^{2} \\
& \text { sur fibre intérieure } \\
\sigma_{t} \text { total }= & 10,58-0,3 \sigma_{\mathrm{FL}}=9,37 \mathrm{~kg} / \mathrm{mm}^{2} \\
& \text { sur fibre extérieure. }
\end{aligned}
$$

TABLEAUX COMPARATIFS

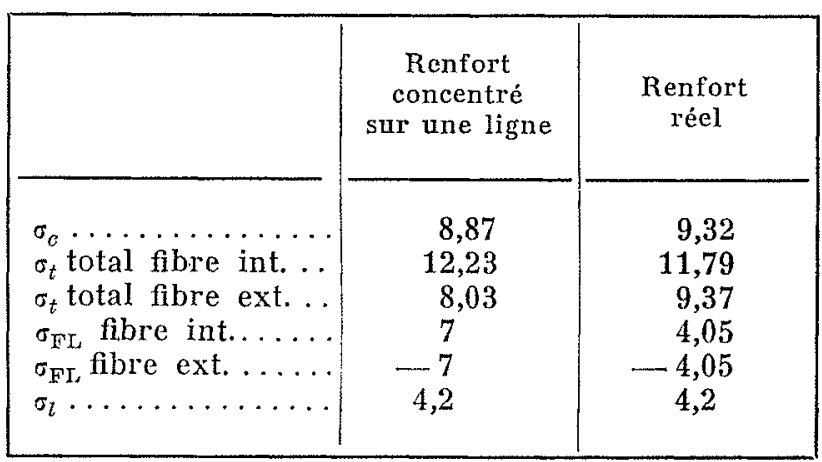

Les contraintes composées correspondantes, d'après le critère de Hencky von Mises, sont respectivement :

$$
\left(\sigma_{e}=\sqrt{\sigma_{1}^{2}+\sigma_{2}^{2}-\sigma_{1} \sigma_{2}}\right)
$$

\begin{tabular}{|c|c|c|}
\hline & $\begin{array}{c}\text { Renfort } \\
\text { concentré } \\
\text { sur une ligne }\end{array}$ & $\begin{array}{c}\text { Renfort } \\
\text { réel }\end{array}$ \\
\hline Sur fibre int. ... & $\begin{array}{c}11,7 \\
9,7\end{array}$ & $\begin{array}{c}10,45 \\
9,3\end{array}$ \\
Sur fibre ext. ... & & \\
\hline
\end{tabular}




\section{D. - ÉTUDE DES TUYAUX FRETTES}

Les tuyaux frettés sont constitués par une paroi intérieure en tôle soudée et par des renforts rapprochés qui sont la plupart du temps des frettes laminées dont les caractéristiques mécaniques sont plus élevées que celles de la tôle de la paroi; par un procédé qui peut consister dans l'enfilage à chaud des frettes sur le tuyau ou bien par le gonflage à froid, par déformation plastique, de la paroi intérieure sur les frettes, on réalise dans l'ensemble frette-paroi un état initial de précontraintes qui crée une tension dans les frettes et une compression dans la tôle.

Les formules précédemment établies s'appliquent à ces tuyaux, mais il est cependant possible de faire certaines simplifications dans leur expression en raison du faible espacement de ces renforts.

\section{D.1 - Tuyaux frettés à chaud.}

Les frettes constituant de tels tuyaux ont souvent la forme ci-dessous; on peut alors considérer que l'interaction frette-paroi se réduit au double système de forces étudiẻ au paragraphe B-2-2.

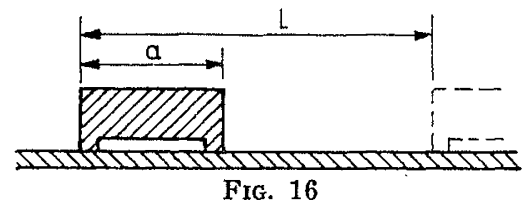

Les formules établies à ce paragraphe sont donc intégralement valables.

Cependant, d'après les valeurs des fonctions $\mathrm{G}$ et $\mathrm{J}_{2}$, on démontre facilement que, dans un domaine de $(a / l)$ compris entre 0,4 et 0,6 (ce qui correspond aux dimensions usuellement employées pour les pas et les largeurs de frettes) et pour des valeurs de $\beta l$ comprises entre 0 et 1,8 ces fonctions se réduisent aux valeurs suivantes à moins de $5 \%$ près :

$\mathrm{G}=\frac{4}{\beta l}$

$\mathrm{J}_{2}=\frac{\beta^{2} l^{2}}{6}\left[3\left(\frac{a}{l}\right)^{2}-3\left(\frac{a}{l}\right)+1\right]=\alpha \frac{\beta^{2} l^{2}}{6}$

De la même manière, les valeurs des fonctions $J$ et $J_{1}$ dans une section au milieu de l'espace entre renforts se réduisent (à moins de $5 \%$ près) à :

$$
\mathrm{J}=\frac{4}{\beta l}
$$

$J_{1}=-\frac{\beta l}{3}\left(1-3 \frac{a^{2}}{l^{2}}\right)=-\alpha_{1} \frac{\beta l}{3}$

Les formules du paragraphe B-2-2 se simplifient alors comme suit, dans les limites que nous venons de préciser :

Si on appelle $e_{f}$ l'épaisseur répartie de la frette sur le pas $l\left[e_{f}=(\mathrm{A} / l)\right]$ et $e_{r}$ l'épaisseur fictive totale $e+e_{f}$, on aura :

$\sigma_{c}=\left\lceil\frac{\mathrm{E} \Delta \mathrm{R}}{\mathrm{R}}-\mathrm{v} \sigma_{l}\right\rceil \frac{e}{e_{r}}+\frac{p \mathrm{R}}{e_{r}}$

au bord d'une frette ou au milieu de l'espace entre frettes :

$\sigma_{t}=\left[-\frac{\mathrm{E} \Delta \mathrm{R}}{\mathrm{R}}+\nu \sigma_{l}\right] \frac{e_{f}}{e_{r}}+\frac{p \mathrm{R}}{e_{r}}$

au bord de la frette :

$\sigma_{\mathrm{F} \mathrm{L}_{\mathrm{L}}}=\left[\frac{\mathrm{E} \Delta \mathrm{R}}{\mathrm{R}}-\mathrm{v \sigma}_{l}\right] \frac{\alpha}{2} \frac{l^{2}}{\mathrm{R} e} \frac{e_{f}}{e_{r}}+p \frac{\alpha}{2}\left(\frac{l}{e}\right)^{2} \frac{e_{f}}{e_{r}}$

au milieu de l'espace entre frettes:

$\sigma_{\mathrm{FL}}=-\left[\frac{\mathrm{E} \Delta \mathrm{R}}{\mathrm{R}}-v_{l}\right] \frac{\alpha_{1}}{4} \frac{l^{2}}{\mathrm{R} e} \frac{e_{f}}{e_{r}}-p \frac{\alpha_{1}}{4}\left(\frac{l}{e}\right)^{2} \frac{e_{f}}{e_{r}}$

$\sigma_{l}$ due à l'effet Poisson $=\frac{v p \mathrm{R}}{e_{r}}$

(d'après le paragraphe B-2-2 en remarquant (que $Z \# 1$ dans les limites précisées).

\section{D.2 - Tuyaux autofrettés.}

La paroi intérieure est fabriquée à un diamètre extérieur de 0,5 à $2 \%$ inférieur au diamètre intérieur des frettes. La paroi, munie de ses frettes, est soumise à une pression hydraulique intérieure soit au moyen d'une presse d'épreuve, soit par l'adjonction de fonds.

L'élévation de la pression fait passer l'ensemble par différents stades : la contrainte équivalente (définie au paragraphe D-2-2) augmente jusqu'à atteindre la limite élastique; la tôle se déforme alors dans le domaine plastique jusqu'à venir en contact avec les frettes. La pression continuant à augmenter, les déformations radiales sont limitées par l'expansion des frettes qui reste dans le domaine élastique, et la contrainte 
dans les frettes équilibre alors la part de pression qui n'est pas équilibrée par la contrainte circonférentielle dans la tôle.

La pression maximale, ou pression de frettage, étant atteinte, on se trouve pour les frettes, dans un état de contrainte élastique constitué uniquement de contraintes circonférentielles, et, pour la tôle, dans un état de contraintes plastique plan (en négligeant les contraintes radiales dues à la pression) dont les composantes principales sont en chaque point une contrainte circonférentielle et une contrainte longitudinale provenant de la flexion élastoplastique entre frettes, augmentée, s'il y a lieu, de la contrainte provenant d'un effet de fond.

La pression diminue ensuite jusqu'à s'annuler et l'ensemble tôle-freltes revient dans le domaine élastique, c'est-à-dire qu'on superpose à l'état de contraintes précédent, l'effet dans le domaine élastique d'une pression négative égale à la pression de frettage.

On retrouve donc, au repos, un état de précontraintes comparable à celui des tuyaux frettés à chaud.

\section{D.2.1. - EXPRESSION DES CONTRAINTES.}

Les frettes utilisées pour les tuyaux autofrettés sont de section rectangulaire pleine. Alors que le frettage à chaud demeure, en principe, dans le domaine élastique, et ne peut de ce fait ramener l'action de la frette sur la tôle à une force unique, l'autofrettage au contraire permet, par la déformation plastique de la tôle sous la frette, une solidarisation totale de ces deux éléments; dans le domaine élastique, l'ensemble tôle-frettes se comporte alors comme un solide isotrope, semblable à un tube crénelé ou décolleté, étant entendu que les modules d'élasticité des frettes et de la tôle peuvent être considérés comme identiques. On peut donc considérer que les frettes agissent sur la tôle comme une série de forces isolées et on est ramené au cas étudié au paragraphe B-1-4.

Cependant, on voit facilement que pour des valeurs de $\beta l$ comprises entre 0 et 1,8 , les fonctions $\mathrm{K}$ et $\mathrm{H}$ sont sensiblement égales à $(2 / \beta l)$, la fonction $\mathrm{H}_{2}$ est égale à $\left(\beta^{2} l^{2} / 6\right)$ tandis que au milieu de l'espace entre deux frettes, la fonction $\mathrm{H}_{1}$ est égale à $(-\beta l / 6)$, à moins de $5 \%$ près.

Si nous désignons par $\sigma_{c_{0}}, \sigma_{t_{0}}$ les précontraintes circonférentielles dans la frette et dans la tôle, et par $\sigma_{F_{L}}$ la précontrainte de flexion entre frettes dans la tôle, nous aurons les expressions simplifiées suivantes :

$$
\sigma_{c}=\sigma_{c_{0}}-v \sigma_{l} \frac{e}{e_{r}}+\frac{p \mathrm{R}}{e_{r}}
$$

au bord d'une frette ou au milieu de l'espace entre frettes :

$\sigma_{l}=\sigma_{t_{o}}+v \sigma_{l} \frac{e_{f}}{e_{r}}+\frac{p \mathrm{R}}{e_{r}}$

au bord de la frette:

$$
\sigma_{\mathrm{FL}}=\sigma_{\mathrm{FL} 0}-\frac{\nu}{2} \sigma_{l} \frac{b^{2}}{\mathrm{R} e} \frac{e_{j}}{e_{r}}+\frac{p}{2}\left(\frac{b}{e}\right)^{2} \frac{e_{f}}{e_{r}}
$$

au milieu de l'espace entre frettes :

$$
\sigma_{\mathrm{FL}}=\sigma_{\mathrm{FL} 0}^{\prime}+\frac{\nu}{4} \sigma_{l} \frac{b^{2}}{\operatorname{R} e} \frac{e_{f}}{e_{r}}-\frac{p}{4}\left(\frac{b}{e}\right)^{2} \frac{e_{f}}{e_{r}}
$$

En vertu de la remarque faite plus haut, il faut, dans les contraintes de flexion, remplacer $l$ par la valeur $b$ représentant l'espace entre bords de frettes.

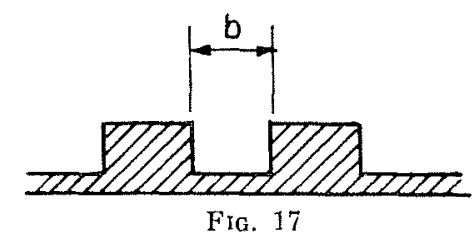

\section{D.2.2 - Comportement AU Frettage Dans LE DOMAINE PLASTIQUE.}

L'étude qui suit est due à $M$. Jean SimonSuisse qui s'est appuyé, pour la théorie de la plasticité, sur "Introduction to the theory of plasticity for engineers," de Hoffmann et Sachs, et sur les travaux de B. Jaoul, du Centre de Recherches de l'École des Mines.

\section{D.2.2.1 - Rappel de la théorie de la plasticité. Définition des domaines.}

Un état de contrainte dont les composantes principales sont $\sigma_{\mathrm{I}}, \sigma_{\mathrm{II}}, \sigma_{\mathrm{III}}$, se trouve dans le domaine ćlastique - et la théorie de l'élasticití est alors applicable - - si le scalaire défini par :

$\sigma_{0}^{2}=\frac{1}{2}\left[\left(\sigma_{I}-\sigma_{11}\right)^{2}+\left(\sigma_{I I}-\sigma_{I I}\right)^{2}+\left(\sigma_{I I I}-\sigma_{I}\right)^{2}\right]$ est inférieur à la limite élastique conventionnelle des matériaux. C'est la théorie de Hencky von Mises. $\sigma_{0}$ est la «contrainte équivalente».

Si la contrainte équivalente $\sigma_{0}$ est supérieure à la limite élastique, l'état de contrainte et dc déformation est soumis aux lois de plasticité.

Si la contrainte équivalente $\sigma_{0}$ est égale à la limite élastique, on est à la frontière de deux théories : domaine élastoplastique.

Pour un acier, la valeur de $\sigma_{n}$ dépend de 
l'écrouissage. Dans un état de contrainte plan $\left(\sigma_{\text {III }}=0\right)$ la contrainte équivalente est :

$$
\sigma_{0}^{2}=\sigma_{\mathrm{I}}^{2}+\sigma_{\mathrm{II}}^{2}-\sigma_{\mathrm{I}} \sigma_{\mathrm{II}}
$$

Dans le domaine de représentation des contraintes d'un état plan de Haig-Westergaard, la frontière entre le domaine élastique et le domaine plastique est une ellipse:

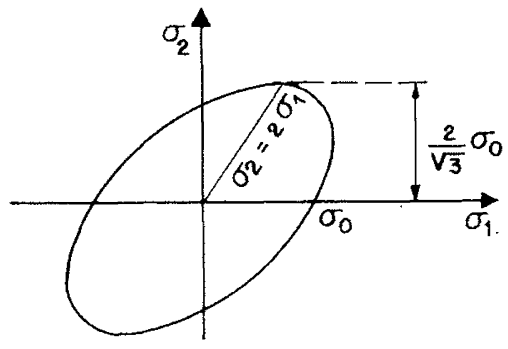

FiG. 18

\section{Théorie de Saint-Venant.}

Dans le domaine plastique, les relations entre contraintes et déformations sont déterminées par la théorie de Saint-Venant, valable pour des matériaux idéalement plastiques:

$$
\left\{\begin{array}{l}
2 \sigma_{\mathrm{I}}-\sigma_{\mathrm{II}}-\sigma_{\mathrm{III}}=6 \lambda \frac{d \varepsilon_{1}}{d t}, \\
2 \sigma_{\mathrm{II}}-\sigma_{\mathrm{III}}-\sigma_{\mathrm{I}}=6 \lambda \frac{d \varepsilon_{2}}{d t}, \\
2 \sigma_{\mathrm{III}}-\sigma_{\mathrm{I}}-\sigma_{\mathrm{II}}=6 \lambda \frac{d \varepsilon_{3}}{d t}, \\
\varepsilon_{1}+\varepsilon_{2}+\varepsilon_{3}=0 .
\end{array}\right.
$$

$\sigma_{I}, \sigma_{I I}, \sigma_{I N I}$, sont les contraintes principales;

$\varepsilon_{1}, \varepsilon_{2}, \varepsilon_{3}$ sont les allongements relatifs dans les directions I, II et III;

$\lambda$ est un paramètre caractéristique $d u$ matériau;

$\frac{d \varepsilon}{d t}$ est Ia dérivée par rapport au temps.

Dans un état plan, $\sigma_{\mathrm{III}}=0$, et on peut écrire ces relations sous la forme:

et

$$
\frac{d \varepsilon_{2}}{2 \sigma_{\mathrm{II}}-\sigma_{\mathrm{I}}}=\frac{d \varepsilon_{3}}{-\sigma_{\mathrm{I}}-\sigma_{\mathrm{II}}}=d \lambda^{\prime}
$$

$$
\varepsilon_{1}+\varepsilon_{2}+\varepsilon_{3}=0 \text {. }
$$

Cette dernière formule exprime les conditions de conservation de volume. Le coefficient de Poisson est de 0,5 .

\section{D.2.2.2 - Détermination des contraintes dans un état plan, la déformation étant bloquée dans une direction.}

Si, à partir d'un état de contraintes $A$, on empêche tout déplacement dans la direction principale I, on peut écrire que $\Delta \varepsilon_{1}=0$, à partir de cet état.

Si l'état A est dans le domaine élastique, on aura d'après la loi de Hooke :

$$
\begin{aligned}
& \Delta \varepsilon_{1}=\frac{1}{\mathrm{E}}\left(\Delta \sigma_{\mathrm{I}}-\nu \Delta \sigma_{\mathrm{II}}\right) \\
& \Delta \sigma_{1}=\nu \sigma_{\mathrm{II}}
\end{aligned}
$$

La représentation dans le plan $\sigma_{\mathrm{I}}, \sigma_{\mathrm{II}}$, est une droite de pente $(1 / \nu)$.

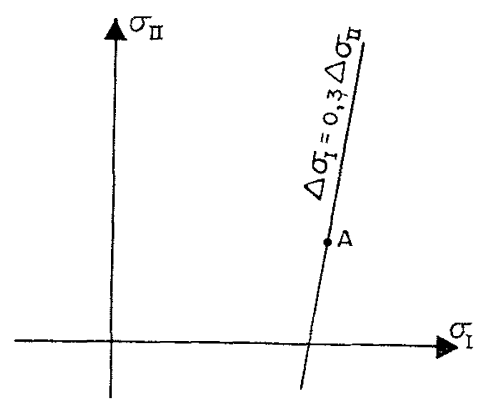

FIG. 19

Si l'état A est dans le domaine plastique, pour un matériau idéalement plastique, c'est-à-dire sans phase élastique, les conditions s'expriment à partir des lois de Saint-Venant :

$$
\Delta \varepsilon_{1}=0 \text { impose } \Delta \varepsilon_{3}=-\Delta \varepsilon_{2}
$$

pour conserver un volume constant.

On a alors :

$$
\frac{-\sigma_{\mathrm{I}}-\sigma_{\mathrm{II}}}{2 \sigma_{\mathrm{II}}-\sigma_{\mathrm{l}}}=\frac{\Delta \varepsilon_{3}}{\Delta \varepsilon_{3}}=-1
$$

Donc :

$$
\sigma_{\mathrm{I}}=\frac{1}{2} \sigma_{\mathrm{II}}
$$

On ne peut donc se déplacer dans le domaine plastique lorsque les allongements dans une direction sont bloqués, que si la contrainte dans la direction bloquée est la moitié de celle existant dans l'autre direction :

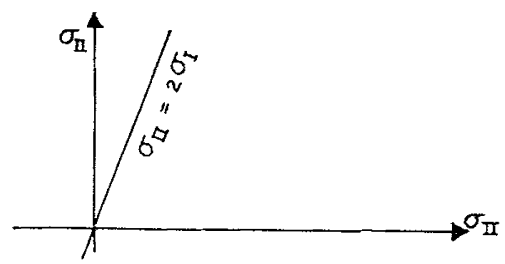

FIG. 20

Ainsi, si dans l'état plastique on part d'un état A qui n'est pas sur la droite $D$

$$
\left[\sigma_{\mathrm{I}}=1 / 2 \sigma_{\mathrm{II}}\right]
$$

il faut, pour qu'il y ait variation des déformations atteindre cette droite $D$, c'est-à-dire que le point représentatif des états successifs va parcourir l'ellipse $\sigma_{0}=$ Cte jusqu'à atteindre la droite $D$, et que tout le long de cette courbe il n'y aura aucune déformation. On dit que l'on reste dans 
un état d'écrouissage équivalent correspondant à $\sigma_{0}$.

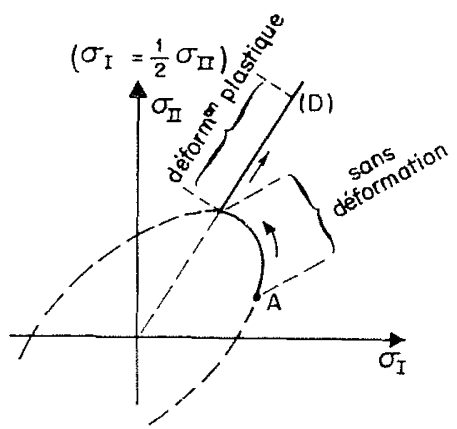

FIG. 21

\section{D.2.2.3. - Application au frettage.}

Lors du frettage, on a évidemment un état de contrainte plan dont une déformation se trouve bloquée par la présence des frettes. Il est bien entendu que l'ellipse $\sigma_{0}$ correspond à l'état d'écrouissage équivalent, que les déformations élastiques des frettes sont petites devant les déformations plastiques de la tôle et que les déformations élastiques dans la phase élastoplastique ne font que transporter l'état de contrainte dans un état plastique très voisin.

-- Frettage entre les plateaux d'une presse :

La seconde contrainte principale provient alors des flexions entre frettes.

A la pression amenant le contact de la tôle sur les frettes, dite pression de collage $p_{c}$, le point figuratif est en $\mathrm{A}$; à partir de ce moment naissent des contraintes $\sigma_{I I}$ de flexion.

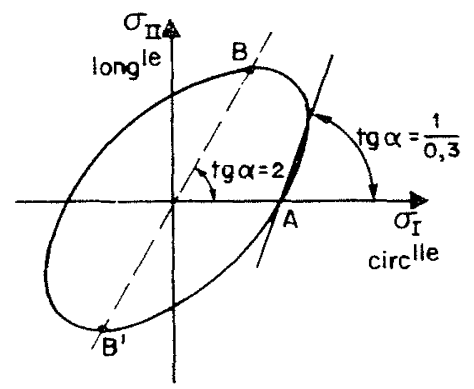

FIG. 22

Pour des $\sigma_{\text {II }}$ positifs, on suivra la droite de pente $(1 / v)$ jusqu'à sa rencontre avec l'ellipse, pour de faibles valeurs de $\sigma_{I I}$, puis l'ellipse ellemême.

Pour des $\sigma_{\mathrm{II}}$ négatifs, on ne pourra que rester sur l'ellipse, puisqu'on ne peut entrer dans le domaine plastique qu'en suivant l'ellipse jusqu'en $B$ ou $B^{\prime}$. Donc, tant que les flexions ne sont pas telles qu'on atteigne $\mathrm{B}$ ou $\mathrm{B}^{\prime}$, on est, au point de vue répartition des contraintes de flexion, dans un domaine identique au domaine élastique. On peut donc écrire que la contrainte de flexion, lorsqu'on a atteint la pression de frettage $p_{f}$, est

$$
\sigma_{\mathrm{FL}}=\frac{1}{2}\left(p_{f}-p_{c}\right)\left(\frac{b}{e}\right)^{2}
$$

formule analogue à celle du paragraphe $D-2-1$ en considérant l'action d'une pression seule, sans précontrainte, cette pression à prendre en compte n'étant que $p_{f}-p_{c}$ puisque la partie $p_{c}$ correspond au collage de la tôle aux frettes.

- Frettage entre fonds :

A la pression de collage, la contrainte longitudinale est moitié de la contrainte circonférentielle et le point figuratif se trouve en $A$. On voit qu'une flexion $\sigma_{\text {II }}$ a tendance à faire sortir de deux côtés dans le domaine plastique, ce qui n'est pas possible tant qu'on n'a pas atteint les points $B$ ou $B^{\prime}$; les points figuratifs se déplacent donc sur l'ellipse. La répartition des contraintes de flexion se trouve donnée encore par la même formule.

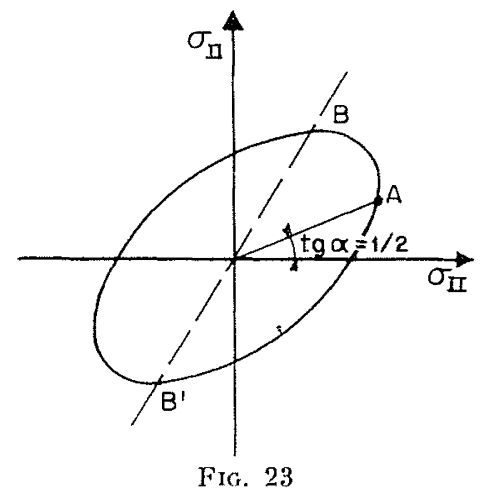

\section{D.2.3 - Calcul des précontraintes et formu- LES DÉFINITIVES.}

Nous nous placerons dans le cas usuel du frettage entre plateaux de presse. Aucun effort longitudinal n'agit donc, en dehors des flexions entre frettes. Si, dans les formules du paragraphe D-2-2 -.. compte non tenu d'un effort longitudinal -, nous portons la valeur de la pression de frettage, nous aurons :

$$
\sigma_{l}=\sigma_{i_{0}}+p_{f} \frac{\mathrm{R}}{e_{r}}=n \%
$$

en appelant $n \%$ la valeur de la limite élastique de la tôle pour un écrouissage de $x \%$. D'où $\sigma_{t_{0}}$.

La valeur de $\sigma_{\epsilon_{\mathrm{o}}}$ s'obtiendra en remarquant que les considérations élémentaires d'équilibre permettent d'écrire :

$$
\sigma_{c_{0}} e_{f}+\sigma_{t_{0}} e=0
$$

Enfin, la valeur de $\sigma_{\mathrm{FL}_{\alpha}}$ s'obtiendra en calculant $\sigma_{\mathrm{FL}}$ pour $p=p_{f}$ soit : ,

$$
\sigma_{\mathrm{FL}_{o}}+\frac{1}{2} p_{f}\left(\frac{b}{e}\right)^{2} \frac{e_{f}}{e_{r}}=\frac{1}{2}\left(p_{f}-p_{c}\right)\left(\frac{b}{e}\right)^{2}
$$

d'où $\sigma_{\mathrm{FL}_{0}}$. 
Finalement, nous aurons les formules générales suivantes, qui tiennent compte de l'action d'un effort longitudinal global $a_{l}$ :

$$
\begin{aligned}
& \sigma_{c}=\left[\frac{p_{f} \mathrm{R}}{e_{f}}-n \% \frac{e_{r}}{e_{f}}-v \sigma_{l}\right\rceil \frac{e}{e_{r}}+\frac{p \mathrm{R}}{e_{r}} \\
& \sigma_{l}=\left[-\frac{p_{f} \mathrm{R}}{e_{f}}+n \% \frac{e_{r}}{e_{f}}+v \sigma_{l}\right] \frac{e_{f}}{e_{r}}+\frac{p \mathrm{R}}{e_{r}} \quad \text { au bord d'une frette ou au milieu de } \\
& \left\{\sigma_{\mathrm{VL}}=\frac{1}{2}\left[\frac{p_{f} \mathrm{R}}{e_{f}}-n \% \frac{e_{r}}{e_{f}}-v \sigma_{l}\right] \frac{b^{2}}{\mathrm{R} e} \frac{e_{f}}{e_{r}}+\frac{p}{2}\left(\frac{b}{e}\right)^{2} \frac{e_{f}}{e_{r}} \quad\right. \text { au bord de la frette; } \\
& \sigma_{\mathrm{FL}}=-\frac{1}{4}\left[\frac{p_{f} \mathrm{R}}{e_{f}}-n \% \frac{e_{r}}{e_{f}}-v \sigma_{l}\right] \frac{b^{2}}{\mathrm{R} e} \frac{e_{f}}{e_{r}}-\frac{p}{4}\left(\frac{b}{e}\right)^{2} \frac{e_{f}}{e_{r}} \text { au milieu de l'espace entre frettes; } \\
& \sigma_{l} \text { due à l'effet Poisson }=\frac{v p R}{e_{r}} \text { (d'après le paragraphe B-1-2). }
\end{aligned}
$$

\section{3 - Exemples relatifs aux tuyaux frettés.}

\section{D.3.1 - TUYAu FRETté a CHAUd.}

Soit un tuyau libre à ses extrémités, $1610 \mathrm{~mm}$ de diamètre intérieur, $e=16$, muni de frettes dont la section A est de $2960 \mathrm{~mm}^{2}$ avec l'espacement indiqué figure 24. La différence des rayons extérieur tôle et intérieur frette, avant frettage est telle que :

$$
\frac{\Delta R}{R}=0,00153 . \text { La pression intérieure est }
$$
$69,15 \mathrm{~kg} / \mathrm{cm}^{2}$.

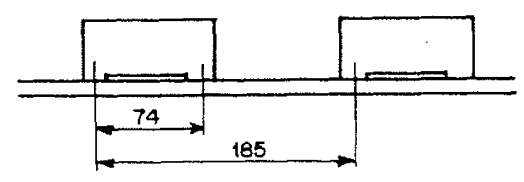

FIC. 24

D'après D-1, nous aurons :

$e_{f}=16 \quad e_{r}=32 \quad \alpha=0,28 \quad \alpha_{1}=0,52 \quad \sigma_{l}=0$

d'où :

$$
\begin{gathered}
\sigma_{c}=15,3+17,4=32,7 \\
\sigma_{t}=-15,3+17,4=2,1
\end{gathered}
$$

au hord d'une frette ou au milien de lespace inter-frettes;

$$
\sigma_{\mathrm{FL}}=4,56+6,46=11,02
$$

atu hord d'une frette;

$$
\sigma_{\mathrm{FL}}=-4,25-6=-10,25
$$

au milıeu de l'espace entre frettes.
Effet Poisson circonférentiel des contraintes de flexion entre frettes:

- au bord d'une frette :

$$
\begin{array}{r}
\sigma_{t} \text { total }=2,1+0,3 \times 11,02=\quad 5,4 \mathrm{~kg} / \mathrm{mm}^{2} \\
\text { sur fibre intérieure; } \\
\sigma_{t} \text { total }=2,1-0,3 \times 11,02=-1,2 \mathrm{~kg} / \mathrm{mm}^{2} \\
\text { sur fibre extérieure; }
\end{array}
$$

- au milieu de l'espace entre frettes:

$$
\begin{array}{r}
\sigma_{t} \text { total }=2,1-0,3 \times 10,25=-0,97 \mathrm{~kg} / \mathrm{mm}^{2} \\
\text { sur fibre intérieure; } \\
\sigma_{t} \text { total }=2,1+0,3 \times 10,25=5,17 \mathrm{~kg} / \mathrm{mm}^{2} \\
\text { sur fibre extérieure. }
\end{array}
$$

Récapitulatif des contraintes totales :

$\sigma_{c}=32,7$

$\sigma_{t}=5,4 \quad$ au bord d'une frette, fibre intérieure

$\sigma_{\mathrm{FL}}=11,2$

$\sigma_{t}=-1,2$

$\gg$

$\sigma_{\mathrm{FL}}=-11,02$

$\sigma_{t}=-0,97$ entre deux frettes, fibre intérieure

$\sigma_{\mathrm{FL}}=-10,25$

$\sigma_{\mathrm{FL}}=5.17$

$\sigma_{\mathrm{FL}}=10,25$ extérieure

extérieure
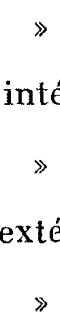


\section{D.3.2. - TUYAU AUTOFRETTÉ.}

Considérons un tuyau autofretté ancré à ses deux extrémités, diamètre intérieur $1450 \mathrm{~mm}$, $e=15$, muni de frettes $80 \times 28$ au pas de 158 . La pression de marche est de $89 \mathrm{~kg} / \mathrm{cm}^{2}$ et la pression de frettage $p_{f}$ de $181 \mathrm{~kg} / \mathrm{cm}^{2}$.

La limite élastique de la tôle après écrouissage est $n \%=36$.

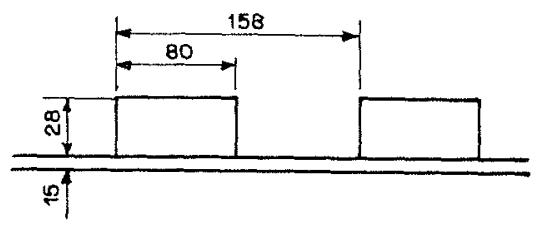

FIG. 25

D'après D-2-3, nous aurons :

$e_{f}=14,2 \quad e_{r}=29,2 \quad b=78$

$\sigma_{l}=6,63 \mathrm{~kg} / \mathrm{mm}^{2}$ dû à l'effet Poisson.

D'où :

$$
\begin{aligned}
& \sigma_{c}=\begin{array}{c}
\text { précontraintes } \\
8,48+22,1
\end{array} \quad=30,56 ; \\
& \sigma_{t}=-8,03+22,1=14,03 \\
& \text { au bord d'une frette ou au } \\
& \text { frettes; } \\
& \sigma_{\mathrm{Fu}}=2,23+5,85=8,08 \\
& \sigma_{\mathrm{FL}}=-1,12-2,92=-4,04 \\
& \text { au milieu de l'espace entre } \\
& \text { frettes. }
\end{aligned}
$$

Effet Poisson circonférentiel des contraintes de flexion entre frettes:

Conformément à ce qui a été exposé au paragraphe D-2-2, le point représentatif, dans la partie tendue par la flexion, peut évoluer dans le domaine élastique sur la droite de pente $1 / v$, tandis que, dans la partie comprimée par la flexion, il évolue sur l'ellipse $n \%$. Du fait qu'on est, en fin de frettage, dans un état élasto-plastique, on ne peut plus appliquer intégralement la règle simple donnée dans l'exemple d'un tuyau fretté à chaud.

$1^{\circ}$ Pour la partie tendue par la flexion, on vérifie, dans cet exemple, qu'on reste sur la droite de pente $1 / \nu$ sans atteindre l'ellipse. On peut alors écrire :

- au bord d'une frette :

$\sigma_{t}$ total $=14,03+0,3 \times 8,08=16,45 \mathrm{~kg} / \mathrm{mm}^{2}$ sur fibre intérieure.

- au milieu de l'espace entre frettes : $\sigma_{t}$ total $=14,03+0,3 \times 4,04=15,24 \mathrm{~kg} / \mathrm{mm}^{2}$ sur fibre extérieure.

$2^{\circ}$ Pour la partie comprimée par la flexion, un calcul complet donne les valeurs suivantes :

- au bord d'une frette :

$$
\sigma_{t} \text { total }=14,03-6,44=\begin{gathered}
7,59 \mathrm{~kg} / \mathrm{mm}^{2} \\
\text { sur fibre extérieure. }
\end{gathered}
$$

- au milieu de l'espace entre frettes: $\sigma_{t}$ total $=14,03-3,15=10,88 \mathrm{~kg} / \mathrm{mm}^{2}$ sur fibre intérieure.

\section{CONCLUSION}

Nous avons vu, au cours de cette étude, l'application d'une théorie générale aux cas particuliers usuels tels que renforts isolés, tuyaux frettés à chaud et autofrettés.

En particulier, la notion de «largeur associée » n'a plus de sens dès qu'on prend en considération des efforts longitudinaux ou des précontraintes, ou bien dès qu'on considère des renforts rapprochés dont les zones d'influence interfèrent.

Par ailleurs, les théories les plus récentes en matière de plasticité permettent d'analyser de près le comportement des tuyaux autofrettés dans les phases de sa fabrication et lors de son fonctionnement sous pression. Sur la base de ce que nous venons d'exposer, on peut étudier en détail le comportement des tuyaux autofreltés dans des cas exceptionnels tels que : diminution de l'épaisseur par corrosion interne, rupture accidentelle d'une ou de plusieurs frettes, pression critique de rupture.

Ces méthodes de calcul permettent, par la meilleure connaissance des phénomènes élastiques et plastiques, d'améliorer le coefficient de sécurité réel des tuyaux et plus spécialement des grandes conduites forcées modernes. 